\title{
Prediction of the Three Dimensional Structure of the DBD of Centromere Protein B (CENP-B) in Comparison with the Structures of Myb, LexA and DtxR DBDs
}

\author{
By Masashi Suzuki, $\left.{ }^{*},+\right)$ Hiroshi Masumoto, ${ }^{* *)}$ and Tuneko OKazaki**) \\ (Communicated by Setsuro EBASHI, M. J. A., May 12, 1995)
}

\begin{abstract}
The centromere protein B (CENP-B) recognises DNA of a particular nucleotide sequence. We have identified two repeating units in the DNA-binding domain (DBD) of CENP-B by examining its amino acid sequence closely. The sequences of the two units resemble not only each other but also the sequences of the DBDs of transcription factors, Myb, LexA and DtxR. Since all the above transcription factors have three $\alpha$-helices in their DNA-binding units, it is likely that each unit of CENP-B is also composed of three $\alpha$-helices. We discuss possible ways in which the two units of CENP-B are combined along the same DNA.
\end{abstract}

Key words : LexA; DNA-binding specificity; molecular recognition; CENP-B; diphteria toxin repressor.

Introduction. The mammalian centromeric heterochromatin has tandem repeats of particular nucleotide sequences. One of these repeats is the $\alpha$-satellite DNA. ${ }^{1,2)}$ By using auto-antibodies which react with the human centromere, three proteins have been located there ${ }^{3)}$-i.e. the centromere proteins (CENPs)-A, B, C. CENP-B binds to the $\alpha$-satellite DNA. ${ }^{4-8)}$ The N-terminal 125 residue domain (the DNA-binding domain, DBD) of CENP-B is sufficient to bind to a 17bp DNA component (the CENP-B box), ${ }^{4,5), 8)}$ and the protein dimerizes via the hydrophobic C-terminal 59 residue domain, which is outside the DBD. ${ }^{9)}$

Two repeating units in the CENP-B DBD. The CENP-B box has a nucleotide sequence, ${ }^{4), 5), 8)} 5$-TTTCGTTGGAAACGGGA-3. It has been shown that the tetramers TTCG, CGGG and an A base between the two (shown in bold) are important for the binding specificity. ${ }^{16)}$ Each CENP-B monomer binds to a CENP-B box and thus a dimer to two such boxes. ${ }^{9)}$ Most of the known DNA-binding proteins use an $\alpha$-helix or a $\beta$-sheet for contacting DNA bases but both

*) MRC Laboratory of Molecular Biology, Hills Road, Cambridge, CB2 2QH, U.K.

**) Department of Molecular Biology, School of Science, Nagoya University, Chikusa-ku, Nagoya 464-01, Japan.

†) Correspondence to: M. Suzuki. an $\alpha$-helix and a $\beta$-sheet can cover essentially only one side of the DNA-covering four to six basepairs at most. ${ }^{10), 11)}$ Since a single CENP-B DBD covers at least 15 basepairs, the DBD appears to have more than one DNA-binding unit, probably two; one binding to the site around TTCG and the other around CGGG.

Following the above assumption, we have examined the amino acid sequence of the CENP-B DBD closely and found that the 125 amino acid residues of the DBD can be divided into two half sites (Fig. 1a). The two halves (RP1 and RP2) resemble each other. Furthermore, these sequences have some resemblance to those of some transcription factors, of which the structures have been determined-i.e. Myb, ${ }^{12)}$ LexA, ${ }^{13)}$ DtxR (diphteria toxin repressor). ${ }^{14)}$ Each of the DNA-binding units of these transcription factors has three $\alpha$-helices and the third helix binds to the DNA. The structures and the modes of DNA-binding of Myb and LexA have been compared in some detail. ${ }^{15)}$

In each of the three helices in a bundle, a few positions facing the same side are predominantly occupied by hydrophobic residues (marked with a and $d$ in Fig. 1b). These hydrophobic residues are important for the packing. RPs 1 and 2 of CENP-B have the same character (Fig. 1b). Also all the real and putative second helices have an Ala residue following the 


\section{a CENP-B DBD

\begin{tabular}{|c|c|c|c|}
\hline & $\begin{array}{l}\text { helix1 } \\
0000000001111111 \\
1234567890123456\end{array}$ & $\begin{array}{l}\text { helix2 } \\
0000000001 \\
1234567890\end{array}$ & $\begin{array}{l}\text { helix3 } \\
00000000011 \\
12345678901\end{array}$ \\
\hline RPI & RQLTFREKSRI IQEVEENP & DLRKGEIARRFNIP & --PSTLSTI LKNKRA I LASE \\
\hline & $1: 1$ & $11: 1$ & $: 1: \quad: 1:: 1: 1$ \\
\hline
\end{tabular}

\section{b Hydrophobic positions \\ helixi \\ helix2 \\ 0000000001111111 \\ 000000004 \\ $1234567890123456 \quad 1234567890$ \\ helix3 \\ 00000000011 \\ 12345678901}

RP 1 RQLTFREKSRIIQEYEENP-DLRKGE IARRENIP--PSTLSTILKNKRAILASE RKYGVASTCRKTNKLSPYD-KLEGLLIAWFQQIRAAGLPVKGIILKEKALRIAE d a d a d a d

MYb-1 TRWTREEDEKLKKLVEONG-TDDWKVIANYLPMR----TDVOCQHRWOKVLNPELIK

MYb-2 GPWTKEEDQRYIKLVQKYG-PKRWSVIAKHLKGR----IGKQCRERWHNHLNPEVKK

MYb-3 TSWTEEEDRI IYQAHKRLG--NRWAE IAKLLPGR----TDNAIKNHWNSTMRRKV

LEXA ARQQQEVEDLIRDHISQTGMPPTRAEIAQRLGER----SPNAAEEHLKALARKG

DEXR LVDTTEMYLRTIYELEEEGVTPLRARIAERLEQS-----GPTYSQTYARMERDG

Fig. 1. Amino acid sequence of the CENP-B DBD (a) in comparison with those of some transcription factors (b). In (a) the same type of residues found at equivalent positions in the two proposed units (RP1 and RP2) are connected by "I", while similar residues by ":". In (b) positions occupied predominantly by hydrophobic residues are marked with a and $d$.
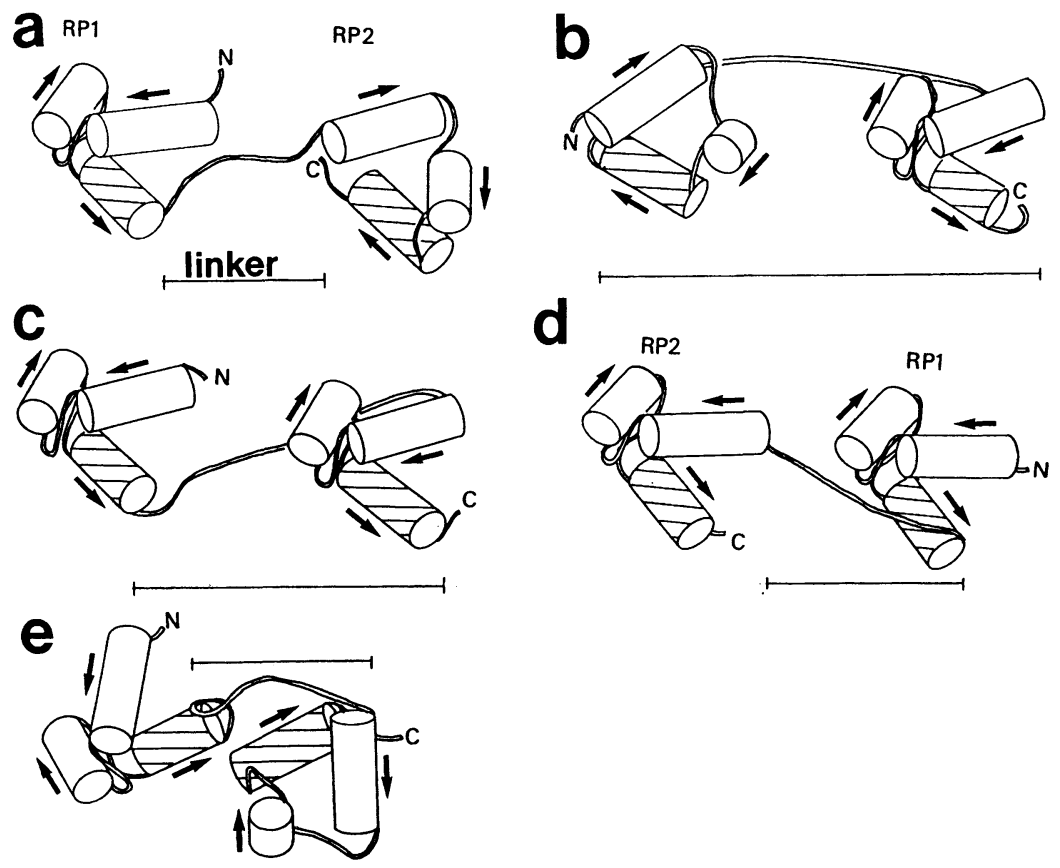

Fig. 2. Models of the CENP-B DBD (a-d) in comparison with the solution structure of the Myb DBD (e). The four models (a-d) are different in the N-C direction (shown by arrows) of the two recognition helices (shown hatched) relative to each other (see also Fig. 3). Compared with the length of the linker (shown by bars) connecting two repeats in $\mathrm{Myb}^{12)}$ (e), that expected for CENP-B in two of the four models appear to be rather long $(b, c)$, while that in the other two similarly short (a, d). RP1 and RP2: the repeating units 1 and 2, respectively, of CENP-B. 
hydrophobic residue, Ile, at the d phase, which seems to be important for this type of packing. Therefore, it seems likely that the two repeats of CENP-B adopt the folding of the three $\alpha$-helix bundle (Fig. 2a).

In earlier work ${ }^{9), 16)}$ we have pointed out that the CENP-B DBD is likely to be $\alpha$-helical. This coinsides with the above discussion, although the number of $\alpha$-helices predicted earlier was four but not six-the four helices correspond to helices 1 and 3 of RP1 and helices 2 and 3 of RP2 discussed in this paper.

Combination of the two DNA-binding units. Since all the transcription factors listed in Fig. 1b use their third helices for DNA recognition (the recognition helices), it seems safe to assume that the two repeats of CENP-B too use their third helices for DNA recognition. If the CENP-B DBD is indeed composed of the two repeating units, regarding the $\mathrm{N}-\mathrm{C}$ direction of the two recognition helices relative to each other, four essentially different ways are possible (Figs. 2, 3). Each of the four has two further choices regarding the direction to which the two units mount on the DNA-i.e. which end of the CENP B-box is closer to, for example, RP1 (Fig. 3). Therefore, altogether eight essentially different modes of CENPB-DNA interaction must be considered (two of the eight modes are shown in Figs. 4b, c).

For understanding which of the four ways is more likely, one of the transcription factors, Myb, is particularly interesting as this has more than one unit and gives us some insight into the ways in which the two units can be linked. We expect that in a likely
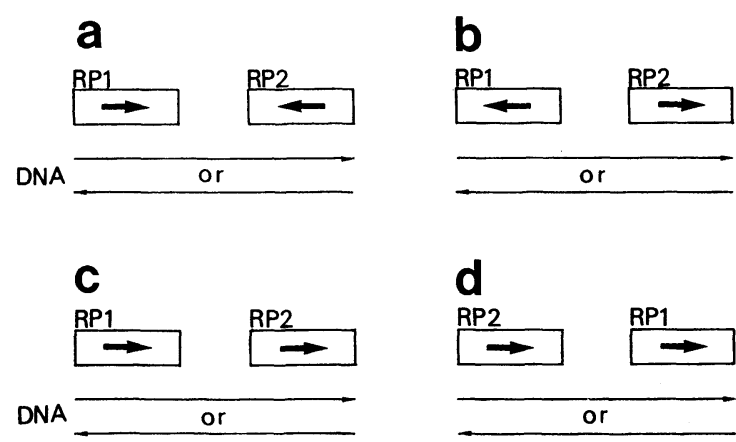

Fig. 3. Schematic presentation of eight different ways of combining two recognition helices of CENP-B along the DNA. Four modes (a-d) are possible regarding the N-C direction (shown by bold arrows) of the two recognition helices (shown as boxes). Each of the four has two further choices regarding the direction of the DNA relative to the protein (shown by thin arrows). Altogether eight different ways for interaction can be created. RP1 and RP2: the repeating units 1 and 2, respectively, of CENP-B. mode of interaction the two units of CENP-B are positioned so that they can be connected by a reasonably short linker when one of the two units is positioned along TTCG, while the other is along CGGG (Figs. $4 b, c)$. By a variety of experiments it has been well established that the two sites directly interact with the protein (ref. ${ }^{16)}$ and Masumoto and Okazaki unpublished, which is summarised in Fig. 4a).

The number of residues found between the two neighboring units in CENP-B seems to be smaller than that in Myb (Fig. 1b). Therefore, the physical length of the CENP-B linker must be similar to or shorter than that of Myb. In comparison with the physical length of the linker found in the solution structure of Myb (Fig. 2e), that expected for CENP-B in two of the four possibilities appears to be rather long (Figs. 2b, c), while that in the other two appears to be similarly
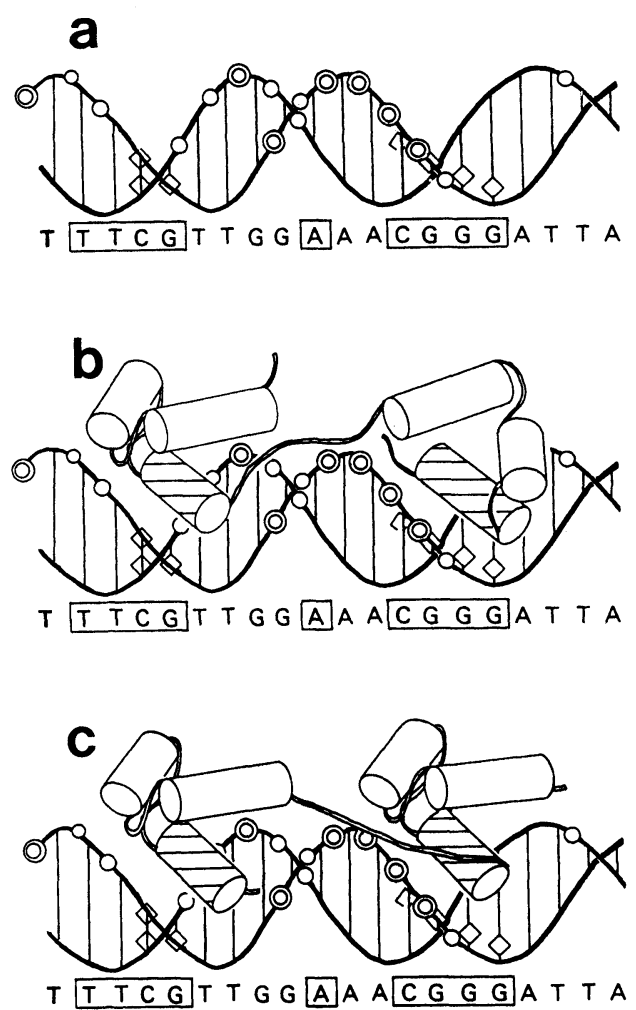

Fig. 4. Features of the DNA recognised by CENP-B (a) and two possible models for CENP-B-DNA interaction (b, c). (a) CENP-B binding was interfered with the ethylation of back bone phosphate (circles and double circles-double circles for stronger interference) and with the methylation of bases (diamonds) (ref. ${ }^{16)}$ and Masumoto and Okazaki, unpublished). (b) and (c) are drawn so that the models of the protein (shown, respectively, in Figs. 2a and d) are placed close to the protected sites on the DNA. Note that the linker crosses over the minor groove near the A base (boxed) which is important for the binding specificity. 


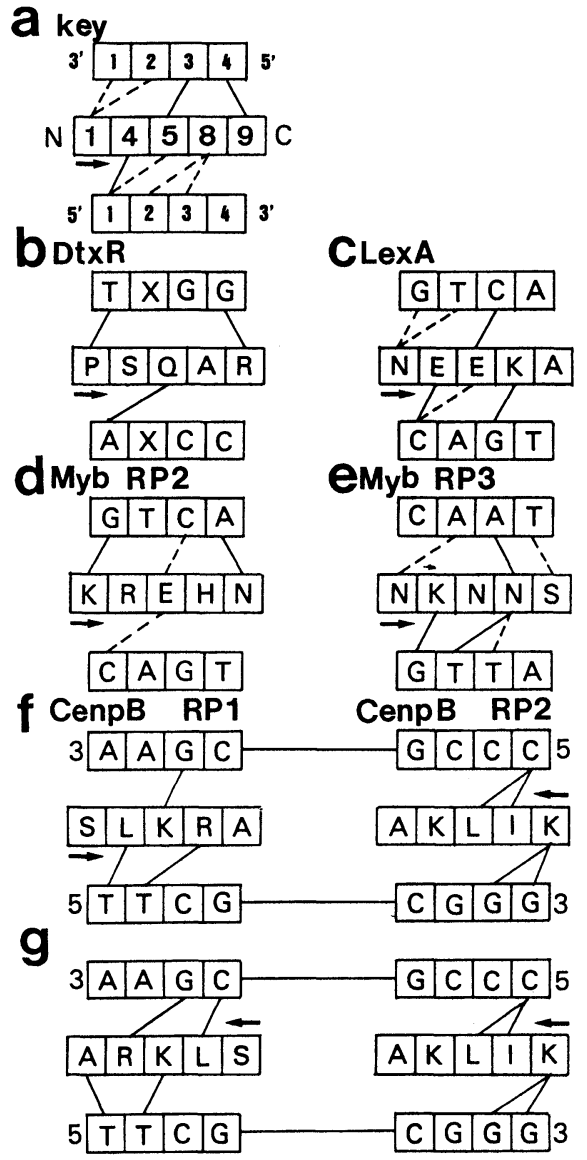

Fig. 5. Possible amino acid-nucleotide base contacts. (a) "Key" for understanding the other diagrams. The five positions in the recognition helix in the center contact bases in the two DNA strands. The diagrams are drawn in the same way as in Fig. 2 of Ref. 15. (b)-(e) The contacts shown for Myb (d, e) are those found in the Myb-DNA complex. ${ }^{12)}$ The LexA and DtxR structures were determined in the absence of DNA and thus the contacts $(b, c)$ are predicted based on some experimental results. ${ }^{14), 15), 17)}$ (f, g) The contacts from CENP-B are purely speculative (see text). (f) corresponds the mode shown in Figs. 2a and $3 \mathrm{a}$, while $(\mathrm{g})$ that shown in Figs. $2 \mathrm{~d}$ and $3 \mathrm{~d}$. In the diagrams of DtxR and CENP-B only the contacts which are specific and thus important are shown. The $\mathrm{N}-\mathrm{C}$ directions of the recognition helices are shown by arrows.

short (Figs. 2a, d, see also Figs. 4b, c), and thus the latter two models seem to be more appropriate.

Possible base-residue contacts. Since we have concluded the two possible recognition helices of CENP-B and the nucleotide sequence of the two binding sites have been experimentally identified, one might expect that now it is possible to predict the contacts made to the DNA bases from amino acid residues in the recognition helices. Unfortunately the available information is not still enough to predict the contacts uniquely.

The first step towards the prediction is to identify the amino acid residues which directly contact the DNA bases. One way of discussing such amino acid residues becomes possible, if one assumes that CENP$\mathrm{B}$ binds to the DNA in the same way as Myb, LexA, and DtxR. This assumption might not be unreasonable as the overall structures of these units are expected to be similar as already discussed. One of us has compared the DNA-binding modes of LexA and Myb and has concluded that the units in these proteins recognise the DNA bases in the same way and thus the base-residue contacts fall into the same pattern ${ }^{15)}$ (Fig. 5). The binding specificity of $\mathrm{DtxR}^{14), 17)}$ too can be explained by using the same pattern of contacts (Fig. $5 b)$. Therefore, one might expect that the two repeats of CENP-B might again use the same pattern. In this pattern positions, H3-1, 4, 5, 9, contact the DNA bases. In Figs. 5f and $g$ we show possible amino acid-DNA base contacts of the CENP-B repeats which best match the pattern, when the repeats bind to the DNA in two of the four possible ways.

Further experimental study is necessary to identify which one of the eight binding modes is closest to the truth and finally identify the amino acid residueDNA base contacts. We hope the discussion given in this paper provides a framework to design and interprete such experiments.

Acknowledgements. We thank Dr. J. Finch for his critical reading of the manuscript. The work of H.M. and T.O. was supported by the grants-in-aid for Scientific Research of Priority Areas from the Ministry of Education, Science and Culture, Japan.

\section{References}

1) Waye, J. S., and Willard, H. F. (1987) Nucl. Acids Res. 15, 7549-7469.

2) Willard, H. F., and Waye, J. S. (1987) Trends Genetics 3, 192-198.

3) Eanshaw, W. C., and Rothfield, N. (1985) Chromosoma 91, 313-321.

4) Masumoto, H., Masukata, H., Muro, Y., Nozaki, N., and Okazaki, T. (1989) J. Cell Biol. 109, 1963-1973.

5) Muro, Y., Masumoto, H., Yoda, K., Nozaki, N., Ohashi, M., and Okazaki, T. (1992) J. Cell Biol. 116, 585-596.

6) Pluta, A. F., Saitoh, N., Goldberg, I., and Earnshaw, W. C. (1992) J. Cell Biol. 116, 1081-1093.

7) Sugimoto, K., Muro, Y., and Himeno, M. (1992) J. Biochem. 111, 478-483.

8) Yoda, K., Kitagawa, K., Masumoto, H., Muro, Y., and Okazaki, T. (1992) J. Cell Biol. 119, 1413-1427. 
9) Kitagawa, K., Masumoto, H., and Okazaki, T. (1995) Mol. Cell Biol. 15, 1602-1612.

10) Suzuki, M., and Yagi, N. (1994) Proc. Natl. Acad. Sci. U.S.A. 91, 12357-12361.

11) Suzuki, M. (1995) Protein Engineering 8, 1-4.

12) Ogata, K., Morikawa, S., Nakamura, H., Serikawa, A., Inoue, T., Kanai, H., Sarai, A., Ishii, S., and Nishimura, Y. (1994) Cell 79, 639-648.

13) Fogh, R. H., Ottleben, G., Rüterjans, H., Schnarr, M., Boelens, R., and Kaptein, R. (1994) EMBO J. 13, 39363944 .
14) Qiu, X., Verlinde, C. L. M. J., Zhang, S., Schmitt, M. P., Holmes, R. K., and Hol, W. G. J. (1995) Structure 3, 87-100.

15) Suzuki, M. (1995) Proc. Japan Acad. 71B, 27-31.

16) Masumoto, H., Yoda, K., Ikeno, M., Kitagawa, K., Muro, Y., and Okazaki, T. (1993) In Chromosome Segregation and Aneuploidy (ed. Vig, B. K.). Springer-Verlag, Berlin, pp. 31-43.

17) Tao, X., and Murphy, J. R. (1994) Proc. Natl. Acad. Sci. U.S.A. 91, 9646-9650. 